\title{
AN ALGORITHM ANAL YZING PHONEME-GRAPHEME AWARENESS THROUGH THE BREAKDOWN OF WORDS
}

\author{
Zachariah Clifford Micallef ${ }^{1}$, Mark Bugeja ${ }^{2}$, Dunstan Briffa ${ }^{1}$ and Dylan Seychell ${ }^{2}$ \\ ${ }^{1}$ Saint Martin's Institute of Higher Education, Schembri Street, Hamrun, HMR 1541, Malta \\ ${ }^{2}$ University of Malta, Msida MSD 2080, Malta
}

\begin{abstract}
Phoneme awareness and orthography are core language skills, in this paper the relationship between both is monitored. There are two main parts to this experiment. The first is a group of algorithms that enable the measures of the described relationship and the second uses the previous measurements to solve a classification problem that related to a real-world problem. Participants are asked to spell a list of words by audio and divide each word into groups of letters that describe the shortest units of sound. This is done so that the correlation between how a person hears a word and how they write it can be observed. Once the data is collected the former part is used to deduct a score. The latter part of the experiment is done to prove the effectiveness of the scoring. Dyslexia is a clinical issue that is known to affect phonological awareness that relates to poor orthography. For this experiment, participants with this profile were asked to participate. Using the score obtained from the data, a classification model is trained in an attempt to classify dyslexic and non-dyslexic participants. This will be used to gauge the effectiveness of the scoring. The experiment has already been proven to work with a limited amount of data.
\end{abstract}

\section{KEYWORDS}

Accessibility, Dyslexic, Analyzing Spelling, Machine Learning, Phonological Awareness, Diagnosing

\section{INTRODUCTION}

When observing a participant's attempt to match each phone (the shortest unit of sound) to a group of letters lead to the understanding one's phoneme to grapheme interpretation of a word. This indicates how text is being interpreted. In this paper, the awareness of how phonemes and graphemes to each other is referred to as phoneme-grapheme awareness. Having a measurement of such a skill can indicate how a language as a whole is being decoded rather than recalled. Creating a scoring method that relates to phoneme-grapheme awareness can indicate that some comprehend sounds relate to spelling more than others. Phoneme-grapheme awareness differs between dyslexics and non-dyslexics. Therefore, a classification model with the application of the scoring method should distinguish dyslexics and non-dyslexics, confirming that the scoring matches a real scenario. Previously this experiment was carried out in a primitive manner since the data was interpreted manually. This consisted of two groups of 8 participants, one consisted of dyslexic participants and the other was a control group. The reason for this was to determine if such a process is useful and effective before developing an automatic algorithm. Positive results were obtained with the use of a KNN, further research was deemed to be useful. Figure 1. Phonological Accuracy Vs Total spelling errors shows the results of the above-mentioned experiment. This paper covers how the computerized algorithm works. Testing is being undertaken on a larger scale with the use of a mobile and desktop application, which was specifically designed for this experiment. 


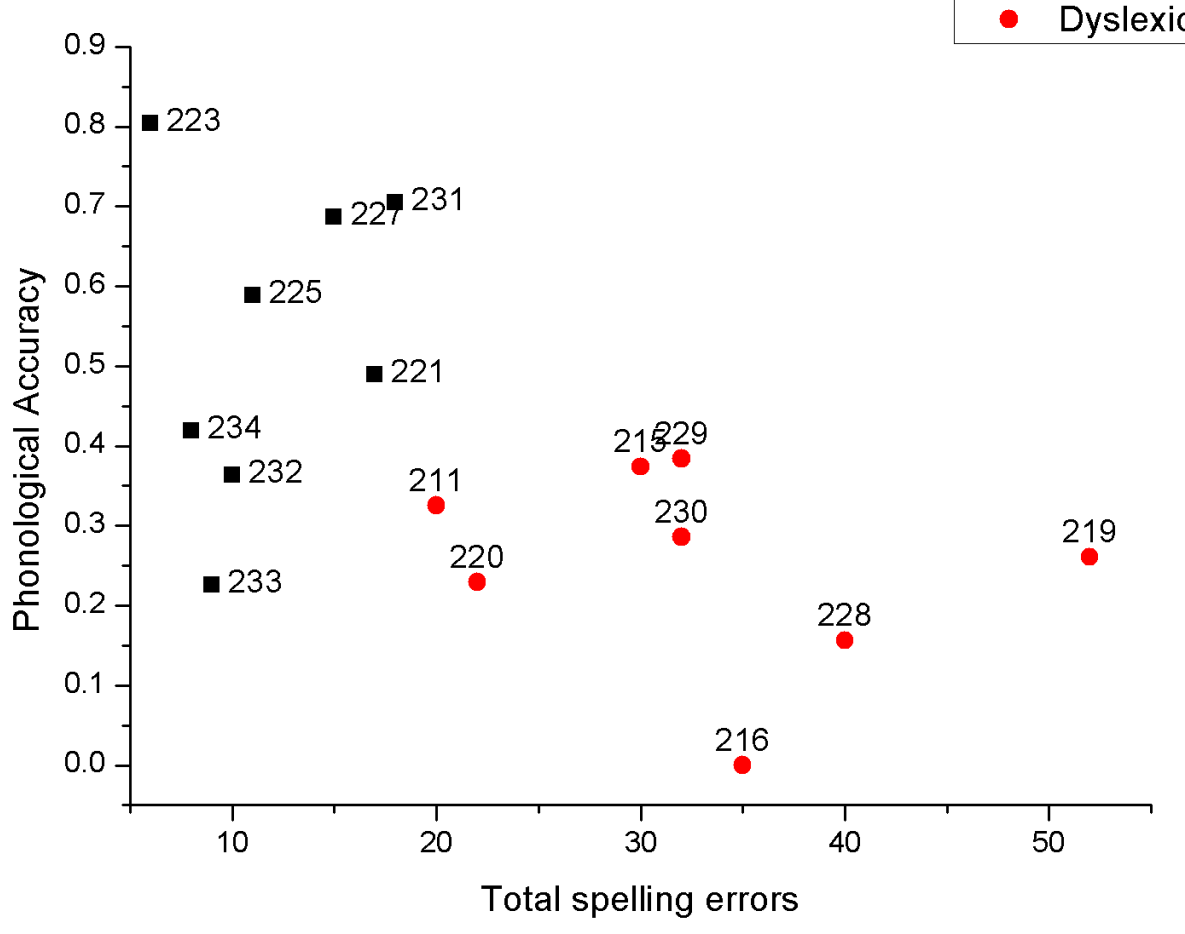

Figure 1. Phonological Accuracy Vs Total spelling errors

\section{BACKGROUND}

\subsection{Dyslexia}

Dyslexia is a neurobiological disorder rooted in the phonological processing system, which impairs the ability to analyze language by word, syllable and sound boundaries. Therefore, a person will have problems with sounding out words, difficulty recognizing known words, poor spelling as well as slow and problematic reading (Sally \& Bennett, 2003). This causes dyslexics to have a natural initial failure to acquire normal word recognition skills and thus reflects poor phonological awareness skills (Maggie, 1992).

\subsection{Phonological Awareness, Graphemes and Phonemes}

Measures of phonological awareness are highly correlated with the ability to read and spell words (Adams, 1990). Phonological awareness is comprised of; performing blending of sounds together, segmenting words into syllables, recombining sounds of words, and judging whether two words have some sounds in common. Phonological awareness also includes skills based on the structure of words such as whether syllables are onsets or rime as well as the distinction of phonemes (Anthony J. L., 2005). The demonstration of this awareness is displayed during the early stages of literacy, where the detection or manipulation of syllables and detection of similar- and dissimilar-sounding words can take place. At higher levels the realisation of onsets and rime, and manipulation of sounds within words is possible. At the highest point of phonological awareness, the individual phonemes within intrasyllabic word units are detected and can be manipulated. Generally combining phonological information is done before words of the same linguistic complexity can be segmented (Anthony J. L., 2003). When analyzing how individual phonemes are produced within alphabetic writing systems, phonological activation corresponds to either a single or multiple graphics units. Thus for example, in English, the letter ' $t$ ' activates the phoneme/t/, and digraph 'sh' activates the phoneme / $/$ / Charles, 1994). 


\subsection{English Orthography}

English is deep in its orthographic depth, this means that the language lacks a consistent grapheme-to-phoneme relationship. Therefore, phonemes and graphemes are subjected to be interpreted in multiple ways that lack clarity. In shallow orthographies, like Finnish, the letters are directly related to their sounds and guided by simple rules (Aro, 2004).

\subsection{Levenshtein Distance}

The Levenshtein distance is the calculation of the minimum amount of changes done from one word (the correct word) to another, one character at a time. Each letter can be inserted, deleted or substituted (Levenshtein, 1965). Measuring the minimum cost sequence of "edit operations" is commonly used as a solution to the string-to-string correction problem. To complete this, the Wagner-Fischer algorithm is used to carry our the Levenshtein distance using a full matrix (Robert \& Michael, 1974).

\subsection{Smith Waterman}

The Smith-Waterman Algorithm is intended to be used for biological sequence alignment to identify commonalities in molecular subsequences as well as solving the problems in molecular analysis. The Smith-Waterman Algorithm measures gaps and similarities this was done at first using an iterative matrix method of calculation, since then other methods were created (Smith \& Waterman, 1981). The algorithm became extremely useful for strings. In order to compare words with there sounded, having gaps and matches just like DNA the Smith-Waterman Algorithm was used. These models are known to be called grapheme-tophoneme (G2P) models (Aaron, et al., 2016).

\section{METHODOLOGY}

The conducted experiment in this research is divided into two groups. The experimental group (dyslexics) and the control group (non-dyslexics). Each participant is required to break down words from oral to written form. Additionally, participants are also required to group the letter or letters that represent the corresponding phone. For example, if the word heard is "sheep", the expected results are groups that contain a letter or multiple letters that relate to the shortest unit of sound such as "sh", "ee" and "p". Participants access an online application that is designed to collect the data for the experiment. Ideally, the participants are required to be over 18 years so that cognition of segmenting words into groups of letters is developed. English is required to be the participant's first language so that the interpretation of the language has more consistency. In this paper, evaluating phoneme-grapheme awareness is based on a score. The score is based on two main axes: spelling, which is measured by the Levenshtein Distance and, the grouping of letters measured by using an altered Smith-Waterman algorithm. This algorithm compares the way the participant group the letters with the theoretical way the word is assumed to be correct. Thus The altered algorithm solution was altered to match the inputted groups of letters entered by the user and compared to the assumed correct grouping of these words. This is done unconventionally by studying the way phonemes and graphemes relate. The altered Smith-Waterman algorithm work by tallying the mistakes found from the comparison in three ways. The first type is failing to isolate a group of letters. This is when a group of letters inputted (attempted by the user) is equal to many groups of letters in the assumed correct counterparts. For example, if the word 'sheep' was interpreted as constituting the groups of letters $x$ ( $x$ being the inputted division of word) having 'shee' and ' $p$ ' and comparing it to its correct counterparts y (y being the assumed correct division of word) having 'sh', 'ee' and 'p', $x[0]$ matches $y[0]+y[1]$ therefore $y[0]$ and $y[1]$ where unsuccessful isolated as groups during the interpretation. The second type is when multiple groups of letters that are inputted (by the user) belong to one group of letters. For example, if the word 'she' was interpreted as constituting groups $x$ having 's', 'h' and 'e' and comparing it to its assumed to be correct counterparts $y$ as 'sh' and 'e'. $x[0]+x[1]$ match the letters of $y[0]$, therefore, $\mathrm{y}[0]$ has been interpreted as a split group rather than the whole. Participants may commit a spelling error but not fail to understand the sound of the word. Words are spelt differently but still produce the same 
sound, such as 'two' and 'too'. The groups of letters such as 'wo' and 'oo' make the /u/ sound, thus having different letters in a group but still produced the same sound. This algorithm takes this into account and the phonemes of a word are obtained from a grapheme-to-phoneme model. These phonemes are displayed as auxiliary symbols as two-letter codes. A sequence of the auxiliary symbols (which make the sounds of the whole world) is also passed into the Smith-Waterman Algorithm so when analysing the groups of letters if an incorrect spelling occurs but still creates the sound, it is not ruled out as incorrect. This brings us to the final type of error when a group has incorrect spelling and does not have any sound correlating. Phoneme-to-grapheme correspondence varies in every language; thus this experiment is tailored for the English language. English is a deep orthographic language and thus orthographically it does not have a consistent one-to-one correspondence between sounds (phonemes) and letters (graphemes). For this experiment, the words chosen have a clear phoneme to grapheme relationship. Once data is processed, the score of phoneme-grapheme awareness is calculated. Using this data, a machine-learning algorithm is trained to classify whether a participant is dyslexic or non-dyslexic. The accuracy of the machine-learning algorithm gives confidence in the phoneme-grapheme awareness scoring system. Judging from the previous smaller excrements a KNN would be best clustering none dyslexics and dyslexics. The flow of data used in the experiment is shown in Figure 2. Data Flow Diagram of Processing the Results.

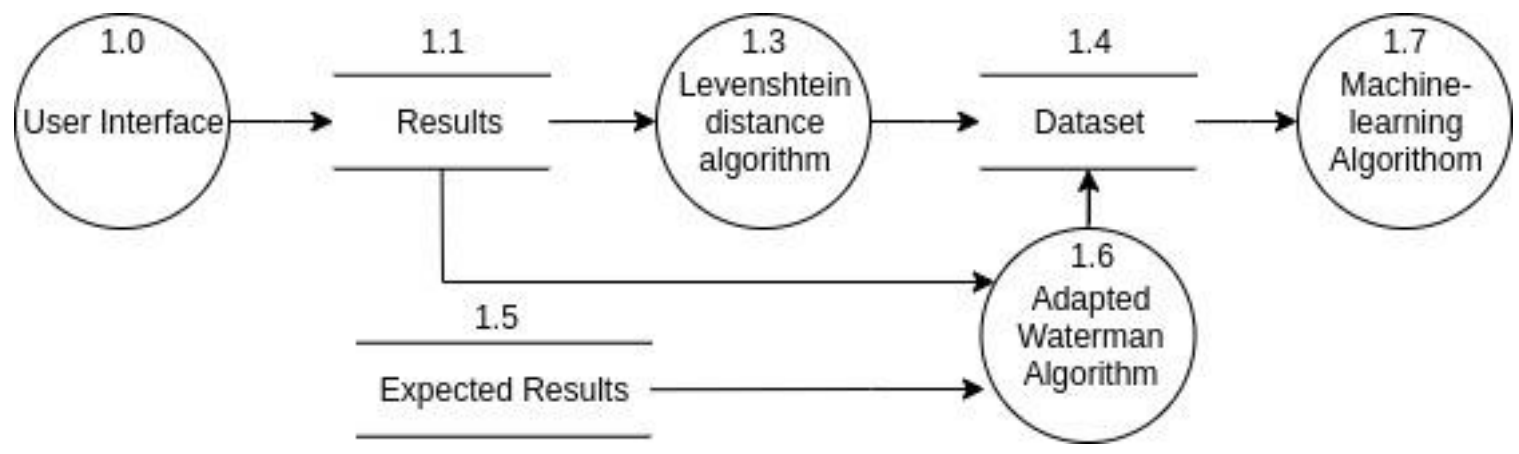

Figure 2. Data Flow Diagram of Processing the Results

\section{FUTURE WORKS}

The research is intended to track how graphemes-to-phonemes are claimed to be related by the participants. From this data, a hypothetical understanding of phoneme-grapheme awareness can be understood to the extent that dyslexia can be identified. The next step for this research is to establish a classification model and test it on a large scale dataset, this includes lots of participants. Furthermore, future work consists of evaluating machine learning algorithms with the potential to classify individuals based upon the models' understanding of the users' difficulties in spelling. This leads to a more robust and responsive intervention, empowering the people with the most common learning disabilities dyslexia and/or specific reading disabilities.

\section{REFERENCES}

Aaron, v. d., Sander, D., Heiga, Z., Karen, S., Oriol, V., Alex, G., . . Koray, K. (2016). WaveNet: A Generative Model For Raw Audio. London: Google DeepMind.

Adams, M. J. (1990). Beginning to read: Thinking and learning aboutprint. Cambridge: MIT Pres.

Anthony, J. L. (2003). ReadingResearch Quarterly. In J. L. Anthony, Phonological Sensitivity: A quasi-parallel progression of word structure units and cognitive operations (pp. 470-487). Hoboken: Wiley-Blackwell.

Anthony, J. L. (2005). Development of Phonological Awareness. Sage Journals, 255 - 256.

Aro, M. (2004). introduction. In M. Aro, Learning to read: The effects of Orthography (p. 9). Jyväskylä: University of Jyväskylä.

Charles, A. P. (1994). Reading in English and Chinese: Evidence for a "Universal" Phonological Principle. In R. Frost, Advances in Psycology Orthography, Phonology, Morphotogy, and Meaning (p. 228). holland: Elsevier Science. 
Kanishka Rao, F. P. (2015). Grapheme-to-phoneme Conversion Using Long Short-term Memory Recurrent Neural Networks. Brisbane: IEEE.

Levenshtein. (1965). Binary Codes Capable of Correcting Deletion, Insertions, and Reversals. Cybernetics and Controle Theory, 707-710.

Maggie, B. (1992). Persistence of Dyslexics' Phonological Awareness Deficits. Developmental Psychology, 874-875.

Robert, A. W., \& Michael, J. F. (1974). The String-to-String Correction Problem. Journal of the Association for Computing Machinery, 168-173.

Sally, E. S., \& Bennett, A. S. (2003). Defining dyslexia, Comorbidity, Teachers' Knowledge. Annals of Dyslexia, 1-14.

Smith, T. F., \& Waterman, M. S. (1981). Identification of Common Molecular Subsequences. Journal of Molecular Biology, 195-197.

Yaoshu, W., Jianbin, Q., \& Wei, W. (2017). Efficient Approximate Entity Matching UsingJaro-Winkler Distance. WISE, 231-239. 